\title{
A uterus didelphys with breech presentation in a previously scarred uterus; an incidental finding
}

Shilpa H. B.*

Department of Obstetrics and Gynecology, Live 100 Hospital Singasandra, Banglore, Karnataka, India

Received: 10 June 2017

Accepted: 08 July 2017

\section{*Correspondence:}

Dr. Shilpa H. B.,

E-mail: drhbshilpa@gmail.com

Copyright: $\odot$ the author(s), publisher and licensee Medip Academy. This is an open-access article distributed under the terms of the Creative Commons Attribution Non-Commercial License, which permits unrestricted non-commercial use, distribution, and reproduction in any medium, provided the original work is properly cited.

\begin{abstract}
A didelphic uterus results from failed fusion of the paired mullerian ducts characterized by two separated uterine horns, each with an endometrial cavity and uterine cervix. Pregnancies develop in one of the two horns, and of the major uterine malformations, the didelphys uterus has the best reproductive prognosis. Improved fetal survival may be secondary to earlier diagnosis, which favors earlier and more intensive prenatal care. Pregnancy is associated with an increased risk of malpresentations and premature labor, although many patients will have no reproductive difficulties. We report a case of successful pregnancy outcome in our institute in a case of didelphys uterus by Caesarean section.
\end{abstract}

Keywords: Breech, Previous caesarean section, Uterus didelphys

\section{INTRODUCTION}

Uterus didelphys occurs when 2 mullerian ducts develop normally but they fail to fuse giving complete duplication of uterine horns and cervices with no communication between duplicated endometrial or endocervical cavities. ${ }^{1}$

There is presence of two separated uterine horns, each with an endometrial cavity and with uterine cervix. A longitudinal vaginal septum is seen in most of the cases. $^{2,3}$ Of the major uterine anomalies, the uterus didelphys has the best reproductive prognosis due to the presence of collateral connections between the two horns which improves the blood supply. It occurs 1 in 5000 to 1 in 15000 women. $^{4}$

Overall, it accounts for about $11 \%$ of the major uterine anomalies.$^{5-7}$ Pregnancies occurring in a didelphys uterus present with malpresentations and preterm labour though there may not be any menstrual or coital difficulties.

\section{CASE REPORT}

A 32-year lady Mrs. XYZ has got admitted to the hospital with a diagnosis of G2P1L1 with 35weeks gestational age with breech presentation with previous LSCS with mild pre-eclampsia with premature rupture of membranes in labour. The first LSCS was done as the patient had a longitudinal vaginal septum. On per abdominal examination, uterus corresponded to 36 weeks size which was relaxed with breech in the lower pole. On per speculum examination, there was a longitudinal vaginal septum with separate cervix on each side of the septum. On per vaginal examination, presence of two cervix was confirmed which was uneffaced and os admitted one finger with clear liqour draining. It was decided to take her for an emergency caesarean section. On opening the abdomen, there was one horn of the uterus with a fallopian tube and ovary and with a single fetus in breech presentation. On the left side, was the non-pregnant uterus lying posterior and to the left side of the pregnant uterus. The non-pregnant uterus had the left fallopian 
tube and the ovary. An alive male baby of $3.1 \mathrm{~kg}$ was extracted from the right horn. There was a single placenta in the right uterine horn. Both the uterine cavities were explored and were found to be separate. There was no post-partum hemorrhage. There were placental bits in the non-pregnant uterine horn likely due to decidual reaction which was sent for histopathology. Histopathology reports confirmed it to be products of conception. The post-operative period was uneventful. Both mother and baby were healthy.

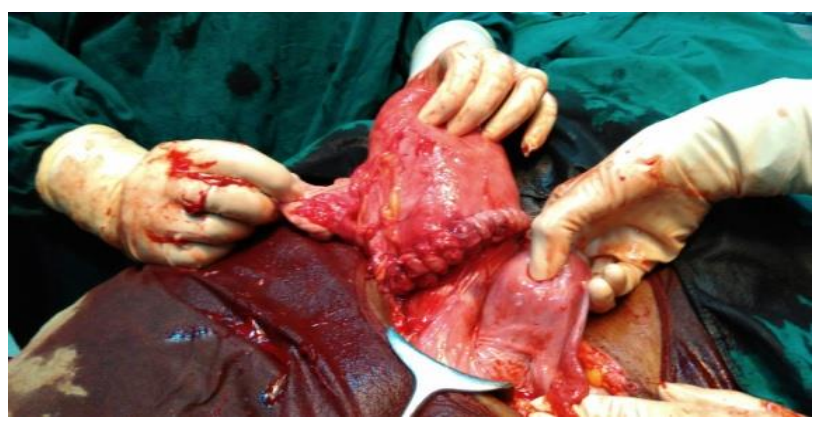

Figure 1: Intaoperative findings showing didelhys uterus. it shows the pregnant right horn of the uterus which is sutured after extraction of the baby and the non-pregnant left horn of the uterus.

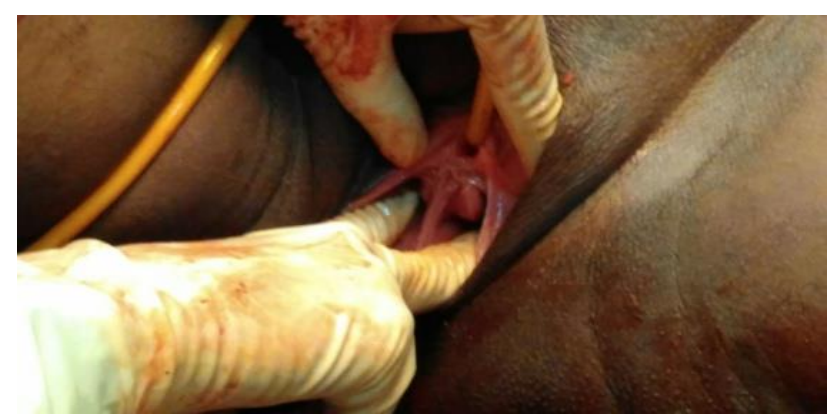

Figure 2: Per vaginal examination showing a vertical vaginal septum.

\section{DISCUSSION}

Uterus didelphys is also called as double uterus. Most of the congenital uterine anomalies lead to malpresentations and preterm labour. Uterine anomalies usually result in a low percentage of viable pregnancies. The pregnancies in a didelphys uterus has better prognosis than those in bicornuate, septate or arcuate uteri. Incidence of singleton pregnancy in uterine didelphys is 1 in 3000 , incidence of twin gestations is 1 in 5 million, incidence of triplets in uterine didelphys is 1 in 25 million.

The detection of uterine anomalies in early pregnancy is of great importance. Sonography has been reported to be useful in identifying abnormal uterine development in most of the cases. Transvaginal sonography offers a new reliable diagnostic method in predicting uterine anomalies in the very early stages of pregnancy.
In the above-mentioned case report, it was possible to detect a uterus didelphys with a viable pregnancy in the right horn only during intra operative period as patient presented very late in third trimester.

In similar case reports by Sudha $\mathrm{R}$ et al from MMC and RI in 2014, Mysore, they presented a successful outcome of an alive $1.9 \mathrm{~kg}$ baby in a didelphys uterus at term. ${ }^{2}$

In another presentation by Jena $\mathrm{L}$ et al, SCB Medical College, Cuttack, presented a successful outcome of a $2.4 \mathrm{~kg}$ male baby extracted by breech at term. ${ }^{3}$

In a similar case report by Rai $\mathrm{M}$ et al from MGM hospital Mumbai in 2014, they presented a single live successful outcome by caesarean section at 29 weeks by breech extraction. ${ }^{1}$

Similar outcome is also seen in our case implying the common complications to be preterm delivery and malpresentations especially breech presentation.

\section{CONCLUSION}

Prevalence of uterine anamolies accounts to $0.1 \%-0.3 \%$. Out of which didelphys uterus accounts to $11 \%$ of all major anamolies. In malformed uterus, complications are increased. Though there is a high incidence of complications associated with uterine anamolies, early detection, good antenatal care, proper counselling and timely intervention by caesarean section helps in achieving favourable outcomes by reducing the complications.

Out of the many complications that have been associated with uterus didelphys, malpresentations and preterm labour are found to be most commonly associated with the condition.

\section{Funding: No funding sources \\ Conflict of interest: None declared \\ Ethical approval: Not required}

\section{REFERENCES}

1. Rai M, Thamke P, Chatterjee A, Kulkurani A. Uterine dydelphys with pregnancy in right cornu. J Evol Med Dental Sci. 2014;3(24):6644-8.

2. Sunanda N, Sudha R, Vineetha R. Second trimester spontaneous uterine rupture in a woman with uterine anomaly: a case report. Int J Sci Stud 2014;2(8):229231.

3. Jena L, Puhan J, Swain S, Kanungo S. (2017). Uterine didelphys: a rare case report. Int J Reprod Contracept Obstet Gynecol. 2017:4(5):1613-4.

4. Elyan A, Saeed M. Müllerian duct anomalies: Clinical concepts. ASJOG. 2004;1:11-20.

5. Carrington BM, Hricak H, Nuruddin RN, Secaf E, Laros Jr RK, Hill EC. Müllerian duct anomalies: MR imaging evaluation. Radiology. 1990;176(3):715-20. 
6. Console D, Tamburrini S, Barresi D, Notarangelo L, Bertucci $B$, Tamburrini $O$. The value of the MR imaging in the evaluation of Müllerian duct anomalies. La Radiologia Medica. 2001;102(4):22632.
7. Brown MA. MR imaging of benign uterine disease. Magnetic Resonance Imaging Clin North Am. 2006;14(4):439-53.

Cite this article as: Shilpa HB. A uterus didelphys with breech presentation in a previously scarred uterus; an incidental finding. Int J Reprod Contracept Obstet Gynecol 2017;6:3706-8. 